\title{
A Reduced-CP Approach to SC/FDE Block Transmission for Broadband Wireless Communications
}

\author{
António Gusmão, Member, IEEE, Paulo Torres, Member, IEEE, Rui Dinis, Member, IEEE, and Nelson Esteves
}

\begin{abstract}
For conventional cyclic prefix (CP)-assisted single-carrier/frequency-domain equalization (SC/FDE) implementations, as well as for orthogonal frequency-division multiplexing (OFDM) implementations, the CP length is known to be selected on the basis of the expected maximum delay spread. Next, the data block size can be chosen to be large enough to minimize the CP overhead, yet small enough to make the channel variation over the block negligible. This paper considers the possibility of reducing the overall $\mathrm{CP}$ assistance, when transmitting sequences of SC blocks, while avoiding an excessively long fast Fourier transform window for FDE purposes and keeping good FDE performances through low-complexity, noniterative receiver techniques. These techniques, which take advantage of specially designed frame structures, rely on a basic algorithm for decision-directed correction (DDC) of the FDE inputs when the $\mathrm{CP}$ is not long enough to cope with the time-dispersive channel effects. More specifically, we present and evaluate a novel class of reduced-CP SC/FDE schemes, which takes advantage of a special frame structure for replacing "useless" CP redundancy by fully useful channel coding redundancy, with the help of the DDC algorithm. When using the DDC-FDE technique with these especially designed frame structures, the impact of previous decisions, which are not error-free, is shown to be rather small, thereby allowing a power-efficiency advantage (in addition to the obvious bandwidth-efficiency advantage) over conventional block transmission implementations under full-length CP. Additionally, the DDC algorithm is also shown to be useful to improve the power efficiency of these conventional implementations.
\end{abstract}

Index Terms-Block transmission, channel coding, cyclic prefix (CP), frequency-domain equalization (FDE), single-carrier (SC) modulations.

\section{INTRODUCTION}

$\mathbf{S}$ INGLE-CARRIER (SC) modulations are known to be suitable for cyclic prefix (CP)-assisted block transmission within broadband wireless systems, since a low-complexity, linear frequency-domain equalization (FDE) technique, involving simple fast Fourier transform (FFT)-based computations, can then be employed to solve the severe intersymbol

Paper approved by N. Al-Dhahir, the Editor for Space-Time, OFDM, and Equalization of the IEEE Communications Society. Manuscript received June 13, 2005; revised October 9, 2005 and June 9, 2006. This paper was presented in part at the IEEE International Symposium on Communication Theory and Application, Ambleside, U.K., July 2005, and in part at the IEEE International Conference on Communications, Istanbul, Turkey, June 2006.

A. Gusmão, P. Torres, and N. Esteves are with CAPS, Instituto Superior Técnico, Technical University of Lisbon, 1049-001 Lisboa, Portugal (e-mail: gus@ist.utl.pt; paulotorres@ist.utl.pt; nelson.esteves@ist.utl.pt).

R. Dinis is with ISR, Instituto Superior Técnico, Technical University of Lisbon, 1049-001 Lisboa, Portugal (e-mail: rdinis@ist.utl.pt).

Digital Object Identifier 10.1109/TCOMM.2007.892463 interference (ISI) problem [1], [2]. As with current orthogonal frequency-division multiplexing (OFDM)-based schemes, the $\mathrm{CP}$ is long enough to cope with the maximum relative channel delay. Therefore, in what concerns the useful part of each received burst, any interblock interference (IBI) is avoided; moreover, the linear convolutions, in the time domain, which are inherent to the time-dispersive channels, become equivalent to circular convolutions, corresponding to multiplications in the frequency domain. In recent papers [3], [4], we considered both an OFDM option and an SC/FDE option for broadband wireless communications. These papers provided overall comparisons of the two options, with the help of selected performance results, which were used to support the suggestion of a mixed solution for future broadband systems: an OFDM option for the downlink and an SC/FDE option for the uplink. Especially with space diversity in the base station (BS) but not in the mobile terminal (MT), the "implementation charge" becomes concentrated at the BS, where increased power consumption and cost are not so critical), concerning both the signal processing effort and, due to the strong envelope fluctuation of OFDM signals, the power-amplification difficulties (see [5] and the references therein regarding the envelope fluctuation issues). In the meantime, other authors came to similar conclusions about the advantages of that mixed OFDM-SC solution, namely in [6], where the idea was deeply investigated and the possibility of a switched mode with software radio was proposed.

For conventional CP-assisted block transmission implementations, either multicarrier (MC)-based or SC-based, the CP length is selected on the basis of the expected maximum delay spread, so as to ensure that it is always greater than the channel memory order. Next, the data block size can be selected to be small enough to make the channel variation over the block negligible. Since the use of a CP reduces both the spectral efficiency and the power efficiency of block transmission schemes, several approaches have been considered to alleviate this problem, mainly in the MC case, i.e., for discrete multitone (DMT)/OFDM applications (see, for example, [7] and the references therein). Regarding the SC/FDE case, other well-known approaches have also been considered for block transmission with no $\mathrm{CP}$, resorting to "overlap-save," frequency-domain, linear signal-processing methods [8], [9]. However (besides the extra complexity, and/or the performance limitations which are inherent to the linear signal-processing assumption), these methods require FFT windows much longer than the channel memory order, so as to avoid significant performance degradations. Therefore, they are not appropriate when the channel is simultaneously strongly time-dispersive (requiring a 
long $\mathrm{CP}$ ) and highly time-varying (requiring an FFT window which cannot be very long). More recently, selected iterative algorithms have been proposed for IBI cancellation and CP reconstruction in both OFDM and SC receivers, so as to deal with reduced-CP block transmission conditions [10], [11].

In this paper, we consider the possibility of reducing the overall CP assistance when transmitting sequences of SC blocks, possibly with low envelope fluctuation (e.g., due to a phase-shift keying (PSK) symbol constellation), while avoiding an excessively long FFT window for FDE purposes. First, we present in Section II a basic algorithm for a decision-directed correction (DDC) of the FDE inputs when the CP is not long enough to cope with the time-dispersive channel effects. This algorithm is equivalent to subsets of time-domain computations, within the iterative algorithms of [10] (OFDM) and [11] (SC and "fast Hadamard transform (FHT)-precoded" SC), which jointly ensure IBI suppression and CP reconstruction. In Sections III and IV, we propose and evaluate low-complexity, noniterative receiver techniques which resort to the above-mentioned basic DDC algorithm and take advantage of specially designed frame structures for reduced-CP block transmission purposes. In this context, the main proposal is the reduced-CP SC/FDE scheme presented in Section IV (a quasi "CP-free" scheme), which replaces "useless" CP redundancy by fully useful channel-coding redundancy. Additionally, in Section V, the DDC algorithm is also shown to be useful to improve performances in conventional, full-length $\mathrm{CP}$ conditions. Section VI concludes the paper with the relevant conclusions and some complementary remarks.

It should be emphasized that the reduced-CP approach presented and evaluated in this paper, requiring specially designed frame structures, does not need an iterative FDE receiver technique. In a recent paper [12], we presented and evaluated an alternative reduced-CP approach, which requires an iterative FDE technique but can be employed with conventional frame structures where all blocks have a reduced CP, below the channel memory order. This latter approach, which employs a "soft version" of the DDC algorithm, can be regarded as involving a combination of ideas presented in [11] and [13].

\section{DDC ALGORITHM FOR SC-BASED BLOCK TRANSMISSION WITH REDUCED CP}

For a length- $L$ channel impulse response (CIR), let us consider the transmission of length- $N$ SC-based data blocks $\mathbf{s}(i)=\left[s_{0}^{(i)}, s_{1}^{(i)}, \ldots, s_{N-1}^{(i)}\right]^{T}\left(s_{n}^{(i)}\right.$ symbol coefficients taken from, e.g., a quaternary (Q)PSK alphabet), with $N>L$. Whenever a length- $L \mathrm{CP}$ is appended to each data block, the length- $N i$ th useful received block can be represented by

$$
\mathbf{y}_{\mathrm{CP}}(i)=\mathbf{H} \mathbf{s}(i)+\mathbf{n}(i)
$$

where $\mathbf{n}(i)=\left[n_{0}^{(i)}, n_{1}^{(i)}, \ldots, n_{N-1}^{(i)}\right]^{T}$ is the $i$ th received noise vector, and $\mathbf{H}$ is the $N \times N$ circulant matrix which describes the channel effects. The entries of this square matrix, given by $h_{j, k}=h_{(j-k) \bmod N}$, are related to the length- $L$ CIR $\left(h_{n}=0\right.$ for $n=L+1, L+2, \ldots, N-1$ ).

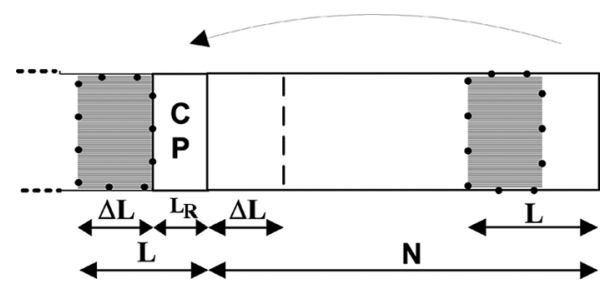

Fig. 1. Reduced-CP block transmission assumptions: $N+L_{R} \geq 2 L$, which is equivalent to $N \geq \Delta L+L\left(\Delta L=L-L_{R}\right)$, ensures that the set of $\Delta L=L-L_{R}$ leading symbols and the set of $L$ trailing symbols, in each length- $N$ data vector, are disjoint.

Let us consider a block transmission under a shortened, length- $L_{R}$ CP, with $0 \leq L_{R}<L$ and $N+L_{R} \geq 2 L$. The initial portion, with $\Delta L=L-L_{R}$ samples, of each received block will differ from the corresponding initial portion under full-length $\mathrm{CP}$, unless

$\Delta s_{j}^{(i)}=s_{j}^{(i)}-s_{j+L_{R}}^{(i-1)}, \quad j=N-L, N-L+1, \ldots, N-L_{R}-1$

is equal to zero. The symbols involved in (2) correspond to the two shadowed areas in Fig. 1. The insufficient CP leads to some IBI and also to an imperfect circular convolution regarding the channel impact on the data block contents.

Obviously, when using $\mathbf{y}(i)$ to denote the new length- $N$ received block, $\mathbf{y}_{\mathrm{CP}}(i)-\mathbf{y}(i)$ will depend on $\Delta s_{j}^{(i)}, j=N-$ $L, \ldots, N-L_{R}-1$. Since $N+L_{R} \geq 2 L$, which is equivalent to $N \geq \Delta L+L$, the set of $\Delta L$ leading symbols and the set of $L$ trailing symbols, in each length- $N$ data vector, are disjoint (see Fig. 1). Therefore, it can be shown that

$$
\mathbf{y}_{\mathrm{CP}}(i)-\mathbf{y}(i)=\mathbf{I}_{\Delta L}^{\prime} \mathbf{H} \mathbf{\Delta}(i)
$$

where

$$
\mathbf{I}_{\Delta L}^{\prime}=\operatorname{diag}[\underbrace{1,1, \ldots, 1}_{\Delta L}, \underbrace{0,0, \ldots, 0}_{N-\Delta L}]
$$

and

$\Delta(i)=[\underbrace{0,0, \ldots, 0}_{N-L}, \underbrace{\Delta s_{N-L}^{(i)}, \ldots, \Delta s_{N-L_{R}-1}^{(i)}}_{\Delta L}, \underbrace{0,0, \ldots, 0}_{L_{R}}]_{(5)}^{T}$.

This means that thanks to $N \geq \Delta L+L$, we get the following relations between the components of the intended received vector $\mathbf{y}_{\mathrm{CP}}^{(i)}$, and the actual received vector $\mathbf{y}^{(i)}$ :

$$
y_{\mathrm{CP}, j}^{(i)}=y_{j}^{(i)}, \text { for } \Delta L \leq j \leq N-1
$$

$y_{\mathrm{CP}, j}^{(i)}-y_{j}^{(i)}={ }^{\prime} j^{\text {th }}$ component of $\mathbf{H} \boldsymbol{\Delta}(i)^{\prime}$, for $0 \leq j \leq \Delta L-1$.

If a perfect a priori knowledge of the $\Delta L$ pairs $\left(s_{j}^{(i)}, s_{j+L_{R}}^{(i-1)}\right), j=N-L, \ldots, N-L_{R}-1$ could be assumed, it should be possible, having in mind (3), to "correct" the received vector $\mathbf{y}(i)$ by replacing it by the appropriate 
$\mathbf{y}(i)$

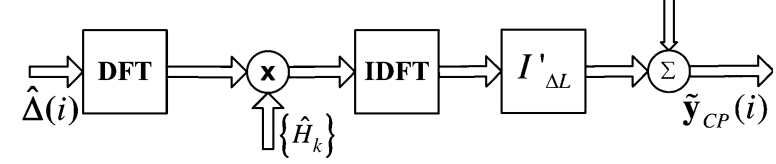

Fig. 2. DDC algorithm implementation.

vector $\mathbf{y}_{\mathrm{CP}}(i)$ prior to FDE. When an estimate of those $\Delta L$ pairs (i.e., an estimate $\widehat{\Delta}(i)$ of $\boldsymbol{\Delta}(i)$ ) is available, a DDC of $\mathbf{y}(i)$ can be carried out, so as to obtain a suitable approximation to $\mathbf{y}_{\mathrm{CP}}(i)$

$$
\begin{aligned}
\tilde{\mathbf{y}}_{\mathrm{CP}}(i)= & \mathbf{y}(i)+\mathbf{I}_{\Delta L}^{\prime} \cdot \mathbf{H} \cdot \widehat{\boldsymbol{\Delta}}(i) \\
= & \mathbf{y}(i)+\mathbf{I}_{\Delta L}^{\prime} \cdot \mathbf{F}^{-1} \operatorname{diag}\left[H_{0}, H_{1}, \ldots, H_{N-1}\right] \\
& \cdot \mathbf{F} \cdot \widehat{\widehat{\Delta}}(i)
\end{aligned}
$$

where $\mathbf{F}$ and $\mathbf{F}^{-1}$ denote a discrete Fourier transform (DFT) matrix and an inverse (I)DFT matrix, respectively, and $\left[H_{0}, H_{1}, \ldots, H_{N-1}\right]^{T}$ is the channel frequency response (CFR) vector, DFT of the CIR vector $\left[h_{0}, h_{1}, \ldots, h_{N-1}\right]^{T}$.

Therefore, a low-complexity, FFT-based algorithm for obtaining $\tilde{\mathbf{y}}_{\mathrm{CP}}(i)$ from the received block $\mathbf{y}(i)$, through the use of the symbol estimates $\hat{s}_{j}^{(i)}$ and $\hat{s}_{j+L_{R}}^{(i-1)}, j=N-L, \ldots, N-$ $L_{R}-1$, can be established as follows (see Fig. 2).

1) Define the vector estimate

$$
\begin{array}{r}
\widehat{\Delta}(i)=[\underbrace{0, \ldots, 0}_{N-L}, \underbrace{\widehat{\Delta s}_{N-L}^{(i)}, \ldots, \widehat{\Delta s}_{N-L_{R}-1}^{(i)}}_{\Delta L}, \underbrace{0, \ldots, 0}_{L_{R}}]^{T} \\
\left(\widehat{\Delta s}_{j}^{(i)}=\hat{s}_{j}^{(i)}-\hat{s}_{j+L_{R}}^{(i-1)}\right) .
\end{array}
$$

2) Compute the DFT of the vector estimate $\widehat{\boldsymbol{\Delta}}(i)$ so as to obtain a frequency-domain vector $\left[\hat{D}_{0}^{(i)}, \hat{D}_{1}^{(i)}, \ldots, \hat{D}_{N-1}^{(i)}\right]^{T}$.

3) Obtain the corresponding frequency-domain vector at the channel output $\left[\hat{D}_{0}^{(i)} \hat{H}_{0}, \hat{D}_{1}^{(i)} \hat{H}_{1}, \ldots, \hat{D}_{N-1}^{(i)} \hat{H}_{N-1}\right]^{T}$, using a CFR estimate.

4) Compute the IDFT of the frequency-domain vector obtained in 3).

5) Retain the initial $\Delta L$ components of the vector computed in 4), and then add them to the initial $\Delta L$ components of $\mathbf{y}(i)$ so as to obtain the vector $\tilde{\mathbf{y}}_{\mathrm{CP}}(i)$, which plays the role of $\mathbf{y}_{\mathrm{CP}}(i)$ in the subsequent FDE procedures.

Of course, one may argue that this algorithm seems useless, since the required set $\left\{\hat{s}_{j}^{(i)} ; j=N-L, \ldots, N-L_{R}-1\right\}$ of symbol estimates, for the current block $i$, is not available before the FDE procedures on this block are carried out. An iterative technique, where increasingly improved estimates of these symbols are obtained and used for DDC-FDE purposes, is an obvious way for taking advantage of the DDC algorithm, when using block transmission schemes with conventional frame structures, but where a reduced CP is adopted in all blocks. This is the approach followed in [12], comparable to that used in [11]. In Sections III and IV of this paper, we propose a different approach, which does not require an iterative receiver implementation, but involves block transmission schemes with special frame structures.

\section{Simple Reduced-CP Block TRANSMission Scheme}

The DDC scheme described in Section II can easily cooperate with a conventional, linear FDE scheme. The FDE coefficients can be given by

$$
F_{k}=\frac{\hat{H}_{k}^{*}}{\hat{\alpha}+\left|\hat{H}_{k}\right|^{2}}
$$

where $\alpha=\sigma_{n}^{2} / \sigma_{s}^{2}$ (with $\sigma_{n}^{2}$ and $\sigma_{s}^{2}$ denoting the variance of noise and data symbols, respectively), provided that widely used linear FDE/minimum mean-squared error (MMSE) results are adopted. The complexity of the DDC scheme is obviously comparable (a bit lower) to that of the conventional FDE scheme, regardless of the values adopted for $N, L$, and $L_{R}<L$, with $N+$ $L_{R} \geq 2 L$ : DFT, frequency-domain multiplication, and IDFT. A block transmission involving CP-free blocks $\left(L_{R}=0\right)$ is just one of the possibilities, which means worst-case conditions for DDC-FDE operation. Especially in this case, a poor $\hat{\Delta}(i)$ estimate can lead to some degradation of the equalization performance; it should be mentioned that the degraded DDC-FDE outputs, which are not uniformly distributed across the FFT window, typically become a bit more significant for the last $L$ symbols.

A simple way of achieving a reduced-CP block transmission, which allows a very simple computation of each required $\widehat{\Delta}(i)$ vector, is depicted in Fig. 3. It consists of alternately transmitting length- $N$ blocks with full-length $\mathrm{CP}$ and length- $(N-L)$ blocks with no CP at all $\left(L_{R}=0\right)$. The FFT window length for FDE purposes is $N$ for both odd and even blocks. Concerning the odd blocks, a conventional FDE procedure is enough for detection. On the other hand, each length- $(N-L)$ even block $i$ (plus the length- $L \mathrm{CP}$ of the odd block $i+1$ ) is submitted to combined DDC-FDE procedures, as explained above. Of course, a previous detection of the odd neighbours $(i-1$ and $i+1)$ of an even block $i$ is required, so as to define $\widehat{\triangle}(i)$ (where $\widehat{\triangle} s_{j}^{(i)}=$ $\left.\hat{s}_{j-N}^{(i+1)}-\hat{s}_{j}^{(i-1)}=\hat{s}_{j}^{(i+1)}-\hat{s}_{j}^{(i-1)}, j=N-L, \ldots, N-1\right)$; this detection can be made through a conventional FDE procedure, since there is a full-length $\mathrm{CP}$ available for all odd blocks.

The following numerical results on SC/FDE uncoded bit-error rate (BER) performance are concerned with broadband wireless transmission over a strongly frequency-selective Rayleigh fading channel, when using the block transmission scheme of Fig. 3, with $N=256$ data symbols per block, each one selected from a QPSK constellation. We consider the power delay profile (PDP) type C within HiperLAN/2, with uncorrelated Rayleigh fading on the different paths. The duration of the useful part of the block is $5 \mu \mathrm{s}$ and the CP has duration $1.25 \mu \mathrm{s}$, corresponding to $L=64$. An invariant channel during each block was assumed, corresponding to a given channel realization, characterized by a randomly selected CIR in accordance with the PDP (maximum delay spread and root mean square (rms) delay spread equal about $1.06 \mu \mathrm{s}$ and $0.15 \mu \mathrm{s}$, repectively). The performance results shown here correspond to an ensemble average over the set of 10000 simulated channel realizations, when a DDC-FDE technique was adopted (for odd blocks, of course, $\mathbf{I}_{\Delta L}^{\prime}=0$ and $\tilde{\mathbf{y}}_{\mathrm{CP}}(i)=\mathbf{y}(i)=\mathbf{y}_{\mathrm{CP}}(i)$, which means conventional 


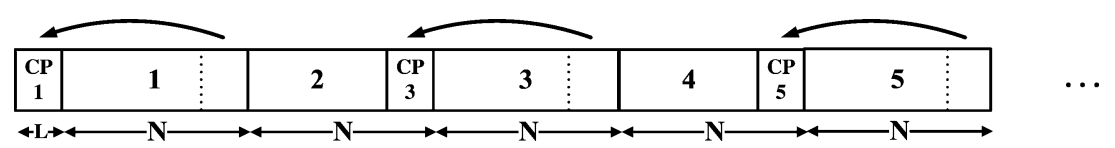

Fig. 3. Simple reduced-CP block transmission scheme.

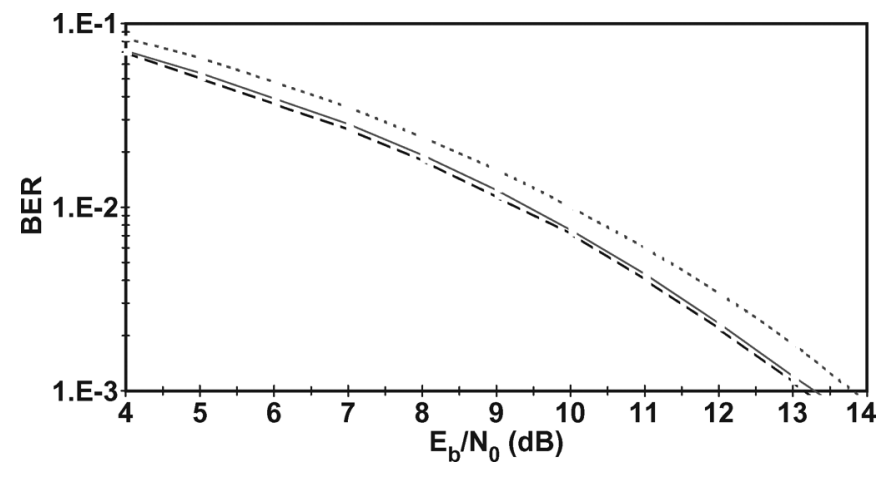

Fig. 4. Uncoded BER performance for a strongly frequency-selective Rayleigh fading channel, with $N=256$ and $L=64$ : odd blocks (dashed line) and even blocks (solid line) of the scheme in Fig. 3; conventional transmission scheme (dotted line).

FDE conditions), and perfect synchronization and channel estimation were assumed. We point out that, for the even blocks in Fig. 3, the DDC-FDE procedures were carried out under worst-case conditions, since these blocks are CP-free.

The simulation results are depicted in Fig. 4, with dashed lines for odd blocks and solid lines for even blocks. These results show that the impact of previous decisions, which are not error-free, on the DDC-FDE receiver performance (for the even blocks) turns out to be negligible, namely for BER $\leq 10^{-3}$.

The block transmission scheme of Fig. 3 requires $2 N$ symbols for an information content which concerns $2 N-L$ symbols ( $N$ as the FFT length and $L$ as the CP length). However, a conventional block transmission scheme with the same FFT length $(N)$ and the same CP length $(L)$, designed for similarly time-varying and time-dispersive channel characteristics, requires $N+L$ symbols for an information content concerning $N$ symbols. This means that, when replacing the conventional transmission scheme by the transmission scheme of Fig. 3, we increase the bandwidth efficiency by $((2 N-L / 2 N) \times(N+L / N)-1) \times 100 \%(9.4 \%$ in our example). The simulation results of Fig. 4 show that thanks to the DDC algorithm, there is no degradation of the power efficiency, and, on the contrary, there is also a power-efficiency advantage over the conventional block transmission implementation. Due to the negligible DDC-related error propagation, this advantage is very close to the ideal advantage which comes from the reduced wasted power concerning the $\mathrm{CP}$ overhead: $10 \times \log _{10}((2 N-L / 2 N) \times(N+L / N))\left(10 \times \log _{10}(35 / 32) \simeq\right.$ $0.39 \mathrm{~dB}$ in our example). Obviously, the transmission scheme which uses length- $N$ FFT blocks for FDE purposes, according to Fig. 3, provides the same bandwidth efficiency as a conventional block transmission scheme designed for the same CP length $L$ and an increased data block length $2 N-L$. Under an ideally time-invariant channel assumption, the power efficiencies should be very similar, through the use of the DDC-aided receiver for the length- $N$ FFT implementation.

Improved ways for taking advantage of the DDC algorithm, in a reduced-CP context, are conceivable, of course. In the following section, we propose a more sophisticated SC/FDE block transmission scheme which only requires $\mathrm{CP}$ assistance in the leading block of a block sequence.

\section{Using ChanNel CODING REDUNDANCY INSTEAD OF CP REDUNDANCY}

\section{A. Code-Assisted SC/FDE Block Transmission Principles}

In the following, we consider the possibility of replacing the conventional CP redundancy, not directly exploited at the receiver side (due to the removal of the corresponding guard period in the received blocks, prior to FDE processing), by a fully useful channel coding redundancy. In the transmission scheme proposed below, which takes advantage of an already available DDC algorithm, we split each length- $N$ coded data block $i$ into two parts: a length- $(N-L)$ "main" block $i$ (with $N \geq L$ ), including some channel coding redundancy, and a length- $L$ "complementary" block $i^{\prime}$, based on the channel coding redundancy not included in the main part.

With a sequence of $M$ two-part coded data blocks $\left(i, i^{\prime}\right)$, the proposed frame is as shown in Fig. 5(a): it requires $M$ contiguous, length- $N$ FFT windows for FDE purposes, but only requires $\mathrm{CP}$ assistance (full-length) in the leading block. The resulting block-transmission efficiency is $\eta=M N /(M N+L)$, which means $\eta \approx 1$ when $M \gg 1$.

For the sake of comparison, Fig. 5(b) shows the corresponding conventional scheme with the same channel coding redundancy, designed for FDE purposes on the basis of $M$ noncontiguous, length- $N$ FFT windows at the receiver side, and leading to a block-transmission efficiency $\eta^{\prime}=N /(N+L)$. When the gross bit rate is the same with the (a) and (b) alternatives, the corresponding data rate $R_{b}$ with the proposed alternative can be significantly higher than the data rate $R_{b}^{\prime}$ using the conventional block-transmission approach. Due to the reduced CP-overhead penalty, $R_{b}=R_{b}^{\prime} M(N+L) /(M N+L)$ $\left(R_{b} \approx(1+L / N) R_{b}^{\prime}=R_{b}^{\prime} / \eta^{\prime}\right.$ if $\left.M \gg 1\right)$.

It should also be noted, as depicted in Fig. 5(c), that a conventional scheme could be aimed at providing about the same data rate $R_{b}$ as the one in Fig. 5(a), with the same data contents per coded block, at the expense of a reduced channel coding redundancy. However, a reduced coding gain, at least for similar decoding complexity, should be expected, together with the increased CP-overhead penalty regarding the power efficiency.

When using the block transmission scheme of Fig. 5(a), the required DDC/FDE/decoding procedures can be carried out as follows. 
A

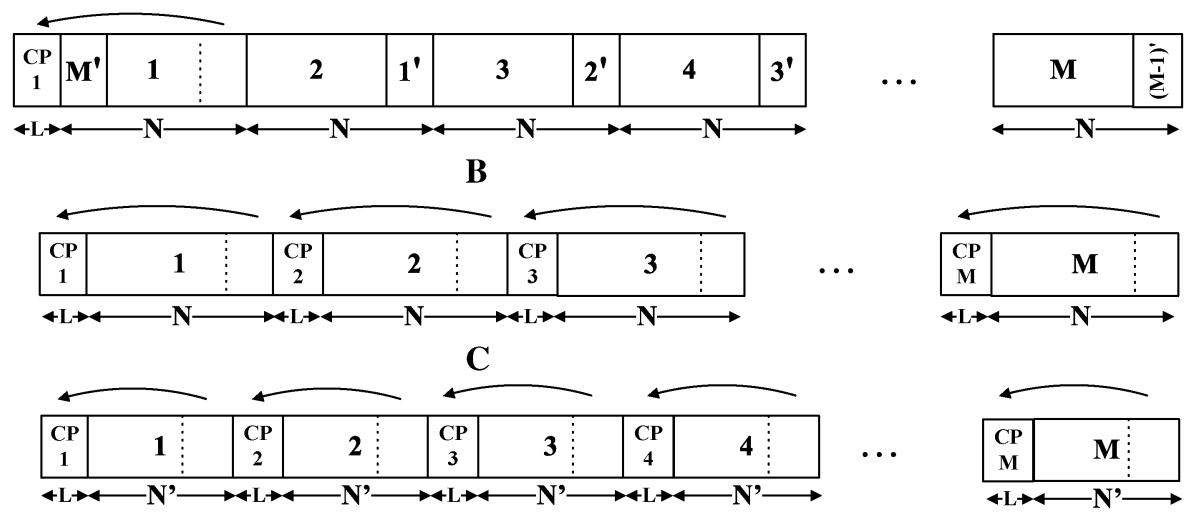

Fig. 5. Highly efficient, code-assisted block transmission scheme (a), and conventional schemes using the same channel coding redundancy (b) and, with $N^{\prime}=$ $N-L$, a reduced channel coding redundancy (c).

\begin{tabular}{|c|c|c|c|c|c|c|c|c|c|c|c|c|}
\hline $\begin{array}{c}\mathbf{C P} \\
1\end{array}$ & M' & 1 & 2 & $1^{\prime}$ & 3 & $2^{\prime}$ & 4 & $3^{\prime}$ & $\cdots$ & $\mathbf{M}$ & $\overline{\bar{I}}$ & $\mathbf{T}$ \\
\hline
\end{tabular}

Fig. 6. Shifted length- $N$ FFT windows for the complementary DDC-FDE procedure (T denotes the length- $L$ required tail, preferably zero-padded).

1) Conventional FDE for the leading block $\left(M^{\prime}, 1\right)$, followed by the decoding of block 1 and a subsequent re-encoding operation, so as to generate an estimate of block $1^{\prime}$ (based on the extra redundancy not included in block 1).

2) [From $i=2$ to $i=M-1$ ] DDC-FDE for block $(i,(i-$ $1)^{\prime}$ ), followed (possibly in parallel) by:

a) decoding of block $\left(i-1,(i-1)^{\prime}\right)$;

b) decoding of block $i$ and subsequent re-encoding, so as to generate an estimate of block $i^{\prime}$.

3) DDC-FDE for block $\left(M,(M-1)^{\prime}\right)$, followed (possibly in parallel) by:

a) decoding of block $\left(M-1,(M-1)^{\prime}\right)$;

b) decoding of block $\left(M, M^{\prime}\right)$.

We point out that the decoding procedures mentioned above can be made simple, since a soft output is not required for the subsequent DDC-FDE operations (but a soft input can be recommendable, for decoding performance reasons).

In Section III, it was reported that under CP-free conditions, a poor estimate of "symbol differences" for DDC-FDE purposes typically leads to an increased degradation of the last $L$ equalized samples. In the context of the proposed code-assisted block transmission scheme, this means that the length- $L$ blocks $1^{\prime}, 2^{\prime}, \ldots,(M-1)^{\prime}$ at the DDC-FDE output typically suffer from worse quality than the equalized blocks $M^{\prime}$ and $1,2, \ldots, M$. Therefore, the subsequent decoding for the $M-1$ pairs $\left(1,1^{\prime}\right),\left(2,2^{\prime}\right), \ldots,\left(M-1,(M-1)^{\prime}\right)$ may not be able to exhibit the same high performance as the $\left(M, M^{\prime}\right)$ decoding, which takes advantage of an equalized block $M^{\prime}$ provided by a conventional FDE operation (hence, not affected by error propagation).

A complementary set of operations, described below, can be carried out so as to solve this error-propagation problem concerning the $M-1$ pairs $\left(1,1^{\prime}\right),\left(2,2^{\prime}\right), \ldots,(M-$
$\left.1,(M-1)^{\prime}\right)$ (a length- $L$ tail, preferably zero-padded, has to be appended to the frame of Fig. 5(a)).

4) [After steps 1-3 described above] DDC-FDE procedures based on the $M-1$ shifted FFT windows shown in Fig. 6, so as to get improved equalized samples regarding the complementary redundancy blocks $1^{\prime}, 2^{\prime}, \ldots,(M-1)^{\prime}$, followed by a new decoding of the resulting paired blocks $\left(1,1^{\prime}\right),\left(2,2^{\prime}\right), \ldots,\left(M-1,(M-1)^{\prime}\right)$ (the equalized samples, obtained previously, concerning the main blocks $(1,2, \ldots, M-1)$ are preserved for decoding procedures).

\section{B. A Coding Design Example and Performance Results}

The ideas of Section IV-A concerning the $\left(i, i^{\prime}\right)$ splitting of each length- $N$ coded data block can easily be made practical by resorting to well-known channel coding schemes. In the design example described and evaluated below, we adopt a rate- $1 / 2$ convolutional code for generating each $\left(i, i^{\prime}\right)$ coded data block: the main part $(i)$ is obtained through a puncturing procedure [14]; the complementary part $\left(i^{\prime}\right)$ is concerned with the redundancy previously removed by that procedure. Many other schemes could be devised, for example based on parallel concatenations of convolutional codes [15]. By using very long coded data blocks and by mapping every block onto several length- $M$ channel block sequences such as that represented in Fig. 5(a) (each sequence assigned to a particular time slot), one could appropriately benefit from the well-known turbo code features.

The BER results shown below are concerned with the SC/FDE block transmission scheme of Fig. 5(a) and to the strongly frequency-selective Rayleigh fading channel already considered in Section II, when $L=64, N=256, M=5$, and a QPSK modulation is adopted (in this case, $\eta=20 / 21$ ). The rate-1/2 convolutional code is an optimum 64-state code, characterized by $g_{1}(D)=1+D+D^{2}+D^{3}+D^{6}$ and $g_{2}(D)=1+D^{2}+D^{3}+D^{5}+D^{6}$, which is punctured to 


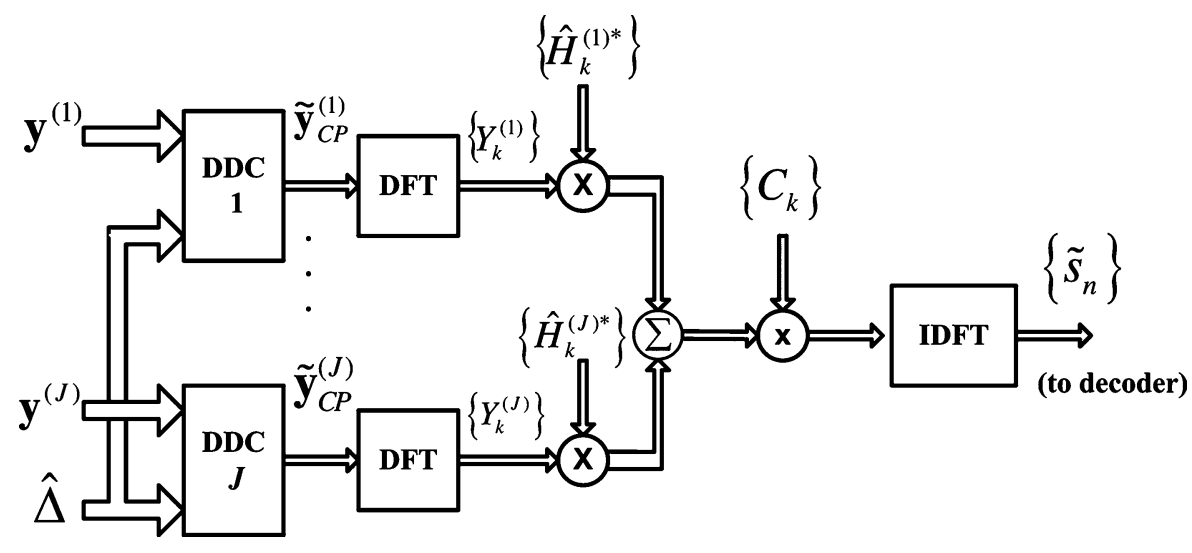

Fig. 7. DDC-FDE receiver with $J$-branch space diversity.

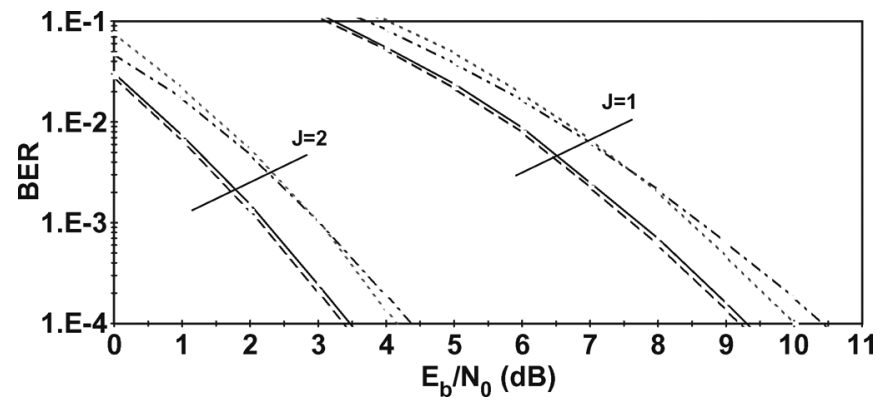

Fig. 8. BER results concerning the block transmission scheme of Fig. 5(a), under the error propagation which is inherent to the DDC procedure when the complementary equalization/decoding "Step 4" is employed (solid lines) or not (dash-dotted lines) and when the simulation assumes an error-free DDC operation (dashed lines) [dotted lines for the conventional transmission scheme of Fig. 5(b)].

obtain a rate- $2 / 3$ code for $\left(i, i^{\prime}\right)$-splitting purposes. The puncturing/splitting procedure is performed according to the matrix

$$
\mathbf{P}=\left[\begin{array}{ll}
1 & 1 \\
1 & 0
\end{array}\right]
$$

where 1 denotes a coded bit for the main part $(i)$, and 0 denotes a coded bit for the complementary part $\left(i^{\prime}\right)$, regarding each of the $M$ paired blocks. With both $i$ and $i^{\prime}$, coded bits are grouped in pairs and mapped onto the QPSK constellation in accordance with a Gray mapping rule.

We also consider the possibility of using more complex receivers, with $J$-branch diversity, when adopting the code-assisted block transmission approach. The required DDC-FDE (MMSE) receiver is shown in Fig. 7 (with $\hat{H}_{k}^{(j)}$ assumed to be employed in each DDC- $j$ processor, $j=1, \ldots, J)$. The equalizer coefficients are as follows [2], [3]:

$$
C_{k}=\frac{1}{\hat{\alpha}+\sum_{j=1}^{J}\left|\hat{H}_{k}^{(j)}\right|^{2}}
$$

with $\alpha$ as defined in Section III (the same noise level is assumed in all receiver branches).

A set of BER results is plotted in Fig. 8. They show that the error-propagation effects which are inherent to the DDC algorithm become negligible, especially for the diversity case, when the complementary equalization/decoding operations (described in "Step 4", in Section IV-A) are employed. Without Step 4, a small performance degradation (about $1 \mathrm{~dB}$ ) can be expected.

By replacing the conventional block transmission scheme of Fig. 5(b) with conventional SC/FDE receiver procedures (e.g., discarding the $\mathrm{CP}$-related samples, etc.) by the proposed block transmission scheme of Fig. 5(a), we obviously increase the bandwidth efficiency by $\left(\left(\eta_{A} / \eta_{B}\right)-1\right) \times 100 \%$, where $\eta_{B}=$ $(N / N+L)$ and $\eta_{A}=(M N / M N+L)$, while keeping the same overall channel coding redundancy. Thanks to the DDC algorithm, which allows a quasi-perfect FDE (negligible error-propagation effects), we can add a power-efficiency gain close to $10 \times \log _{10}\left(\eta_{A} / \eta_{B}\right) \mathrm{dB}$ to the bandwidth-efficiency gain, as shown in Fig. 8. When $N=256$ and $L=64$, as in our example, this means an achievable bandwidth-efficiency gain of about $25 \%$, and simultaneously, a power-efficiency gain of about $1 \mathrm{~dB}$, provided that $M \gg 1$ (about $19 \%$ and $0.76 \mathrm{~dB}$, respectively, when $M=5$ ).

\section{USING CP REDUNDANCY IN CONVENTIONAL BLOCK TRANSMISSION SYSTEMS}

It should be noted that the $\mathrm{CP}$ redundancy, when using a conventional SC/FDE block transmission scheme, can also be regarded as a special kind of channel coding redundancy. In conventional SC/FDE schemes, in general, this redundancy is only used to ensure IBI-free transmission and a channel impact on each data block, which can be described by a circular convolution. The corresponding guard-period samples are discarded at the receiver side, becoming useless for decoding. For a length- $N$ uncoded data block and a length- $L \mathrm{CP}(L \leq N)$, the amount of CP redundancy corresponds to that of $L(2,1)$ repetition codes concerning the last $L$ symbols of the data block (the remaining $N-L$ symbols of the data block are transmitted just as uncoded symbols). When assuming an additive white Gaussian noise (AWGN) channel, an optimum decoding could easily be carried out by considering, for each length- $N$ data block, all $N+L$ samples at the decoder input, including those concerning the length- $L$ CP. $L$ additions of samples corresponding to repeated symbols should be enough for fully exploiting the transmitted CP redundancy at the receiver side. In this context, the 


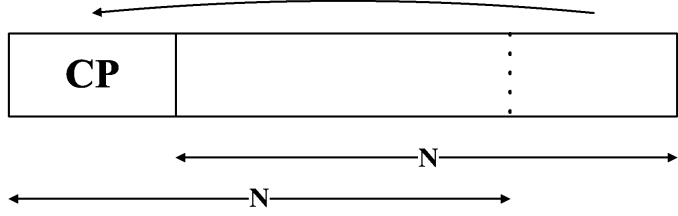

Fig. 9. Length- $N$ FFT windows for fully exploiting the $\mathrm{CP}$ redundancy in conventional SC-based block transmission schemes.

assumption of a QPSK modulation and an optimum synchronization should lead to a BER performance given by

$$
\begin{aligned}
\mathrm{BER} & =\frac{L}{N} \times Q\left(\sqrt{\frac{d_{1}^{2}}{2 N_{0}}}\right)+\frac{N-L}{N} \times Q\left(\sqrt{\frac{d_{2}^{2}}{2 N_{0}}}\right) \\
& =\left(\frac{1}{\eta}-1\right) \times Q\left(\sqrt{\frac{d_{1}^{2}}{2 N_{0}}}\right)+\left(2-\frac{1}{\eta}\right) \times Q\left(\sqrt{\frac{d_{2}^{2}}{2 N_{0}}}\right)
\end{aligned}
$$

with $\eta=N /(N+L), d_{1}^{2}=8 \eta E_{b}$, and $d_{2}^{2}=4 \eta E_{b}$. This leads to

$$
\mathrm{BER}=\left(\frac{1}{\eta}-1\right) Q\left(\sqrt{4 \eta \frac{E_{b}}{N_{0}}}\right)+\left(2-\frac{1}{\eta}\right) Q\left(\sqrt{2 \eta \frac{E_{b}}{N_{0}}}\right) .
$$

The first and second terms on the right-hand side of (11) concern the $L$ repeated symbols and the $N-L$ non-repeated symbols, respectively, for each length- $N$ data symbol block. The ideal QPSK performance on the AWGN channel, BER $=Q\left(\sqrt{2 E_{b} / N_{0}}\right)$, could be achieved with $\eta=1(L=0$, i.e., no repetitions) or $\eta=1 / 2$ ( $L=N$, i.e., all symbols repeated). By discarding the $\mathrm{CP}$ contribution prior to the decision procedures, the optimum BER performance should be given by

$$
\mathrm{BER}=Q\left(\sqrt{2 \eta \frac{E_{b}}{N_{0}}}\right)
$$

for any $\eta$, which means a certain degradation if compared with the BER performance given by (11) (about $0.25 \mathrm{~dB}$ for $L=$ $N / 4$ at BER $=10^{-3}$, for example).

With regards to the frequency-selective wireless channels which are the reason for using a CP-assisted block transmission, it may seem difficult to take advantage of the $\mathrm{CP}$ redundancy at the receiver side, instead of discarding it. However, the DDC-FDE approach proposed here provides an efficient way for a fully useful CP-assisted block transmission (e.g., in accordance with Fig. 5(b), after the required training blocks). For this purpose, we can proceed as follows, taking into account the guard-period samples $y_{-L}^{(i)}, \ldots, y_{-1}^{(i)}$, and also taking advantage of a provisional estimate $\hat{\mathbf{s}}(i)$ on the current block, and the final estimate $\hat{\mathbf{s}}(i-1)$ on the preceding block.

1) Compute a "shifted block" $\tilde{\mathbf{y}}_{\mathrm{SH}}(i)$ (see Fig. 9), given by

$$
\begin{aligned}
\tilde{\mathbf{y}}_{\mathrm{SH}}(i)= & {[\underbrace{y_{-L}^{(i)}, \ldots, y_{-1}^{(i)}}_{L}, \underbrace{y_{0}^{(i)}, \ldots, y_{N-L-1}^{(i)}}_{N-L}]^{T} } \\
& +\mathbf{I}_{L}^{\prime} \cdot \mathbf{F}^{-1} \cdot \operatorname{diag}\left[\hat{H}_{0}, \ldots, \hat{H}_{N-1}\right] \cdot \mathbf{F} \cdot \hat{\boldsymbol{\Delta}}^{\prime}(i) \\
\triangleq & {\left[\tilde{y}_{\mathrm{SH},-L}^{(i)}, \ldots, \tilde{y}_{\mathrm{SH}, N-L-1}^{(i)}\right]^{T} }
\end{aligned}
$$

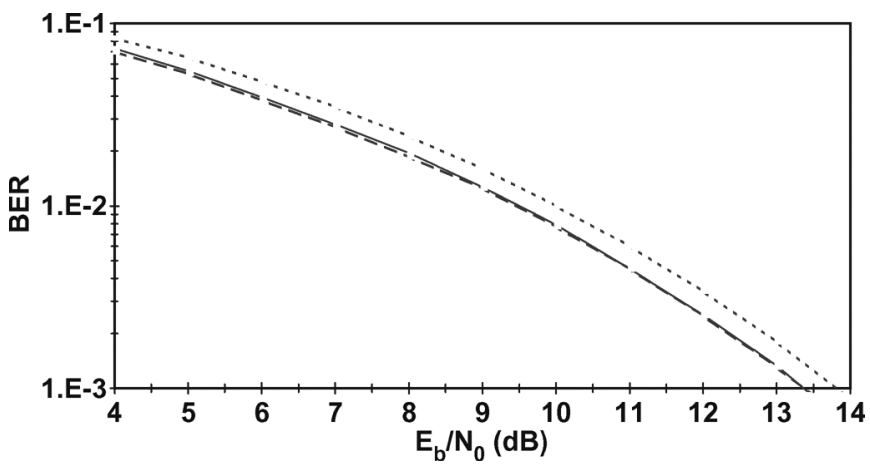

Fig. 10. BER results when the $\mathrm{CP}$ content is used as a simple form of channel coding redundancy: under the error propagation which is inherent to the DDC procedure (solid lines); when the simulation assumes an error-free DDC operation (dashed lines). The conventional BER performance result, when discarding the CP-related samples, is also included (dotted line).

with

$\hat{\boldsymbol{\Delta}}^{\prime}(\mathbf{i})=[\underbrace{0, \ldots, 0}_{N-L}, \underbrace{\hat{s}_{N-2 L}^{(i)}-\hat{s}_{N-L}^{(i-1)}, \ldots, \hat{s}_{N-L-1}^{(i)}-\hat{s}_{N-1}^{(i-1)}}_{L}]^{T}$.

(This computation is a DDC similar to that described in Section II, for the shifted block of Fig. 9.)

2) Define

$\tilde{\mathbf{y}}_{\mathrm{IMP}}(i)=[\underbrace{y_{0}^{(i)}, \ldots, y_{N-L-1}^{(i)}}_{N-L}$,
$\underbrace{\frac{1}{2}\left(y_{N-L}^{(i)}+\tilde{y}_{\mathrm{SH},-L}^{(i)}\right), \ldots, \frac{1}{2}\left(y_{N-1}^{(i)}+\tilde{y}_{\mathrm{SH},-1}^{(i)}\right)}_{L}]^{T}$.

3) Use $\tilde{\mathbf{y}}_{\mathrm{IMP}}(i)$, instead of $\mathbf{y}_{\mathrm{CP}}(i)$, for obtaining a new (and improved) data block estimate.

Fig. 10 shows BER performance results for the conventional scheme of Fig. 5(b), again with $L=64, N=256$, a QPSK modulation, and the channel considered in Section III. In this figure, we show both BER results when the CP contents is used as (a simple form of) channel coding redundancy, and BER results under the conventional FDE procedure, where the CP-related samples are discarded. A small gain can be observed, as expected, when taking advantage of the $\mathrm{CP}$ redundancy, through the use of the DDC-FDE scheme.

\section{CONCLUSIONS AND COMPLEMENTARY REMARKS}

In this paper, devoted to SC/FDE block transmission, we considered the possibility of reducing the overall $\mathrm{CP}$ assistance when transmitting sequences of SC blocks, while avoiding an excessively long FFT window for FDE purposes. The proposed receiver techniques, which take advantage of block transmission schemes with specially designed frame structures, rely on a simple, FFT-based algorithm for decision-directed correction (DDC) of the FDE inputs, when there is no $\mathrm{CP}$ or the $\mathrm{CP}$ is not long enough to cope with the time-dispersive channel effects. 
When using these low-complexity, noniterative receiver techniques with those special frame structures, the error-propagation effects which are inherent to the DDC procedures were shown to be rather small. Therefore, a power-efficiency advantage (in addition to the obvious bandwidth-efficiency advantage) over conventional block transmission implementations under full-length $\mathrm{CP}$ was shown to be achievable. In this paper, the DDC algorithm was also shown to be useful to improve performances within conventional CP-assisted SC/FDE systems, since it enables the exploitation of the $\mathrm{CP}$ redundancy as a special kind of channel coding redundancy.

Further work on synchronization and channel-estimation issues, concerning schemes in accordance with Fig. 5(a), is still required. It seems that adaptive schemes capable of tracking fast channel variations should be based on an explicit channel estimation (for both DDC and FDE purposes), differently from the approach adopted in [16].

Certainly, each channel block sequence, according to Fig. 5(a), should be transmitted after a few training blocks, and the channel estimators of the receiver should be switched to a decision-directed mode (based on symbol estimates) after having processed those training blocks. Anyway, with regard to fast time-varying channel conditions, the transmission scheme of Fig. 5(a) has an obvious advantage over conventional block transmission schemes [Fig. 5(b) and (c)]: it allows high block transmission efficiency $(\eta \approx 1)$ while keeping $N / L$ low enough to ensure quasi-invariant channel conditions across each FFT window for FDE purposes. The proposed approach is clearly recommendable for jointly quite time-dispersive and time-varying channel conditions. In fact, this is an appropriate proposal for facing the designer's dilemma with regards to $\mathrm{CP}$ overhead and channel variation across the FFT window.

Other topics of interest for further research include specific applications to Beyond 3rd Generation cellular systems. Special attention should be devoted to possible adaptations of the code-assisted block transmission approach proposed here to SC-based alternatives using nonlinear modulations with constant or quasi-constant envelope, and allowing good power-bandwidth tradeoffs and low-complexity implementations [17], [18].

The proposed approach seems to be especially interesting for broadband wireless systems designed to operate at the millimetric waveband, with full mobility and significant transmission range. The reasons are clear: for frequencies above $30 \mathrm{GHz}$, full mobility means strong Doppler effects, and, therefore, very fast time-varying channel conditions; for those frequencies, a significant transmission range requires the use of amplifiers with high power efficiency (e.g., grossly nonlinear), hardly compatible with OFDM modulation schemes, since the intended mobility precludes the use of high-gain antennas with low complexity for improving the power budgets.

\section{REFERENCES}

[1] H. Sari, G. Karam, and I. Jeanclaude, "An analysis of orthogonal frequency-division multiplexing for mobile radio applications," in Proc. IEEE Veh. Technol. Conf., Jun. 1994, vol. 3, pp. 1635-1639.
[2] A. Gusmão, R. Dinis, and N. Esteves, "On frequency-domain equalization and diversity combining for broadband wireless communications," IEEE Trans. Commun., vol. 51, no. 7, pp. 1029-1033, Jul. 2003.

[3] A. Gusmão, R. Dinis, J. Conceição, and N. Esteves, "Comparison of two modulation choices for broadband wireless communications," in Proc. IEEE Veh. Technol. Conf., May 2000, vol. 2, pp. 1300-1305.

[4] P. Montezuma and A. Gusmão, "A pragmatic coded modulation choice for future broadband wireless communications," in Proc. IEEE Veh. Technol. Conf., May 2001, vol. 2, pp. 1324-1328.

[5] R. Dinis and A. Gusmão, "A class of nonlinear signal processing schemes for bandwidth-efficient OFDM transmission with low envelope fluctuation," IEEE Trans. Commun., vol. 52, no. 11, pp. 2009-2018, Nov. 2004.

[6] D. Falconer, S. Ariyavisitakul, A. Benyamin-Seeyar, and B. Eidson, "Frequency domain equalization for single-carrier broadband wireless systems," IEEE Commun. Mag., vol. 40, no. 4, pp. 58-60, Apr. 2002.

[7] C. Park and G. Im, "Efficient DMT/OFDM transmission with insufficient cyclic prefix," IEEE Commun. Lett., vol. 8, no. 9, pp. 576-578, Sep. 2004.

[8] E. Ferrara, Jr., "Frequency-domain adaptive filtering," in Adaptive Filters, C. F. Cowan and P. Grant, Eds. Englewood Cliffs, NJ: Prentice-Hall, 1985.

[9] D. Falconer and S. Ariyavisitakul, "Broadband wireless using single carrier and frequency domain equalization," in Proc. Int. Symp. Wireless Pers. Multimedia Commun., Oct. 2002, vol. 1, pp. 27-36.

[10] D. Kim and G. Stüber, "Residual ISI cancellation for OFDM with applications to HDTV broadcasting," IEEE J. Sel. Areas Commun., vol. 16, no. 8, pp. 1590-1599, Oct. 1998.

[11] T. Hwang and Y. Li, "Iterative cyclic prefix reconstruction for coded single-carrier systems with frequency-domain equalization (SC-FDE)," in Proc. IEEE Veh. Technol. Conf., Apr. 2003, vol. 3, pp. 1841-1845.

[12] A. Gusmão, P. Torres, R. Dinis, and N. Esteves, "A class of iterative FDE techniques for reduced-CP SC-based block transmission," in Proc. IEEE 4th Int. Symp. Turbo Codes, Related Topics, Apr. 2006, Paper 71, Session 13.

[13] M. Tüchler and J. Hagenauer, "Linear time and frequency domain turbo equalization," in Proc. IEEE Veh. Technol. Conf., May 2001, vol. 2, pp. 1449-1453.

[14] J. Cain, G. Clark, and J. Geist, "Punctured convolutional codes of rate (n-1)/n and simplified maximum likelihood decoding," IEEE Trans. Inf. Theory, vol. IT-25, no. 1, pp. 97-100, Jan. 1979.

[15] C. Berrou, A. Glavieux, and P. Thitimajshima, "Near Shannon limit error-correcting coding and decoding: Turbo codes," in Proc. IEEE Int. Conf. Commun., May 1993, vol. 2, pp. 1064-1070.

[16] M. Clark, "Adaptive frequency-domain equalization and diversity combining for broadband wireless communications," IEEE J. Sel. Areas Commun., vol. 16, no. 8, pp. 1385-1395, Oct. 1998.

[17] A. Gusmão, V. Gonçalves, and N. Esteves, "Transmission modelling of ENCAP-OQPSK radio links," Inst. Elect. Eng. Proc. Commun., vol. 145, no. 6, pp. 419-425, Dec. 1998.

[18] K. Murota and K. Hirade, "GMSK modulation for digital mobile radio telephony," IEEE Trans. Commun., vol. COM-29, no. 7, pp. 1044-1050, Jul. 1981.

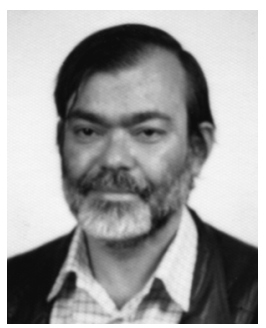

António Gusmão (M'06) received the Ph.D. degree from the Instituto Superior Técnico (IST), Technical University of Lisbon, Lisbon, Portugal, in 1987.

Since 1987, he has been a Professor with IST, with both teaching and research activities in the digital communications area, on which he has published several tens of journal and conference papers. He has also been a member of the Digital Communications Group of CAPS, IST, and the leader of that group since 1998. Since 1992, he has been involved in European projects concerning the development of broadband wireless communication systems. His main research interests concern modeling and simulation of communication systems, modulation and coded modulation issues, and signal processing for broadband wireless communications. 


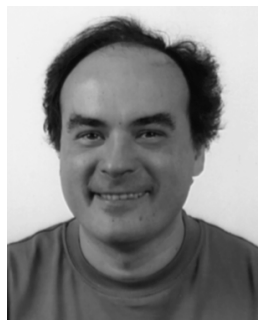

Paulo Torres (M'05) received the M.Sc. degree from the Instituto Superior Técnico (IST), Technical University of Lisbon, Lisbon, Portugal, in 1996.

He has been a member of the Digital Communications Group of CAPS, IST since 1995. Since 1995, he has also been a Professor at the Polytechnic Institute of Castelo Branco, Castelo Branco, Portugal. He has been involved in several research projects in the broadband wireless communications area. His main research interests topics include modulation, equalization, and channel coding.

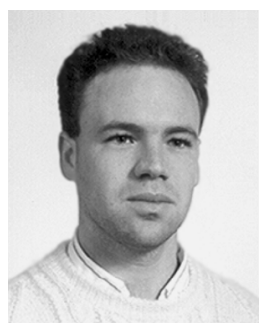

Rui Dinis (S'96-M'00) received the Ph.D. degree from the Instituto Superior Técnico (IST), Technical University of Lisbon, Lisbon, Portugal, in 2001.

Since 2001, he has been a Professor at IST. He was also a member of the Digital Communications Group of CAPS, IST from 1992 to 2005. Since 2006, he has been a Researcher at the Signal and Image Processing Group of ISR, IST. He has been involved in several research projects in the broadband wireless communications area. His main research interests topics include modulation, equalization, and channel coding.

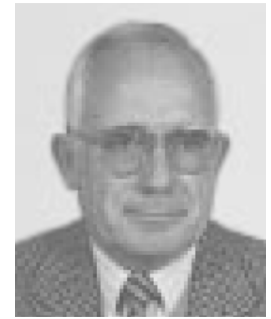

Nelson Esteves received the diploma degree in electrical engineering from the Instituto Superior Técnico (IST), Technical University of Lisbon, Lisbon, Portugal, in 1965, and the Ph.D. degree from Imperial College, University of London, London, U.K., with a dissertation on digital communication, in 1975.

From 1975 to 1979, he was an Assistant Professor at IST, where he was appointed Associate Professor in 1979, and Full Professor in 1989. He has taught in the areas of circuits and signals, telecommunication systems, communication theory, and digital communication. His research interests are in the areas of adaptive signal processing and mobile radio systems. 